Supporting Information for:

\title{
Structure of Light-Harvesting Aggregates in Individual Chlorosomes
}

\author{
Lisa M. Günther ${ }^{1}$, Marc Jendrny ${ }^{1}$, Erik A. Bloemsma ${ }^{2}$, Marcus Tank ${ }^{3}$, Gert T. Oostergetel ${ }^{4}$, \\ Donald A. Bryant ${ }^{3,5}$, Jasper Knoester ${ }^{2}$, Jürgen Köhler ${ }^{1}$
}

\footnotetext{
${ }^{1}$ Experimental Physics IV, University of Bayreuth, D-95440 Bayreuth, Germany

'Zernike Institute for Advanced Materials, University of Groningen, Nijenborgh 4, 9747 AG Groningen, The Netherlands

${ }^{3}$ Department of Biochemistry and Molecular Biology, The Pennsylvania State University, University Park, PA 16802 USA

${ }^{4}$ Groningen Biomolecular Sciences and Biotechnology Institute, Nijenborgh 7, 9747 AG Groningen, The Netherlands

${ }^{5}$ Department of Chemistry and Biochemistry, Montana State University, Bozeman, MT 59717 USA
}

\section{Contents}

1. Ensemble Spectra

2. Movie of the Fit

3. Additional Excitation Spectra

4. Correlation of Energy Splittings

5. Cryo-electron microscopy

6. Simulations / Model Calculations

7. Variations of the Model Structure

8. References 


\section{Ensemble Spectra}

Fluorescence-excitation spectra from ensembles of chlorosomes from WT Cba. tepidum and from the $b c h R$ mutant at different temperatures.

a)

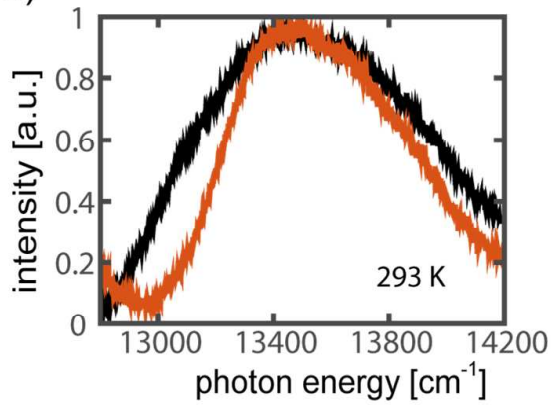

b)

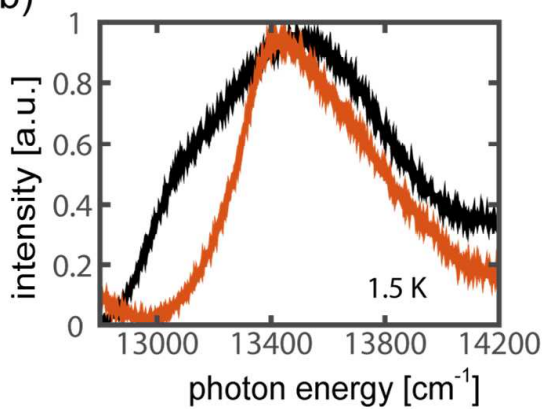

c)

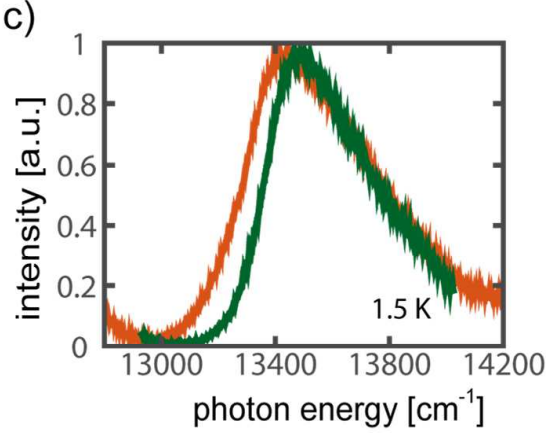

Figure S1: Comparison of ensemble spectra from wild type chlorosomes (black) and chlorosomes from the $b c h R$ mutant (red). (a) Room temperature spectra ( $F W H M_{W T}=990 \mathrm{~cm}^{-1}, F_{W H M}=730 \mathrm{~cm}^{-1}$ ), (b) Low temperature spectra $(1.5 \mathrm{~K})\left(\mathrm{FWHM}_{\mathrm{WT}}=900 \mathrm{~cm}^{-1}, \mathrm{FWHM}_{\mathrm{R}}=540 \mathrm{~cm}^{-1}\right.$ ), (c) Comparison of the low temperature ensemble spectrum from the $b c h R$ mutant (red) with the corresponding sum spectrum (green) from 58 individual chlorosomes $\left(\mathrm{FWHM}_{\text {sum }}=434\right.$ $\mathrm{cm}^{-1}$ ). 


\section{Movie of the Fit}

Link to a movie that illustrates the decomposition of the polarization-resolved fluorescenceexcitation spectrum of an individual chlorosome into four spectral components. The experiments were conducted at $1.5 \mathrm{~K}$.

The left hand side corresponds to the $2 \mathrm{~d}$ representation of the polarization-resolved fluorescenceexcitation spectrum, the right hand side displays the spectra at the current position of the cursor as it progresses through the $2 \mathrm{~d}$ pattern. The noisy black curve represents the experimental spectrum, the coloured lines correspond to the spectral components of the decomposition, and the black smooth line illustrates the sum of the four spectral components. 


\section{Additional Excitation Spectra}

Examples of additional polarisation-resolved fluorescence-excitation spectra from chlorosomes of the $b c h R$ mutant from $\mathrm{Cba}$. tepidum recorded at $1.5 \mathrm{~K}$. Parameters that can be extracted from these particular spectra are summarized in table S1.

a)

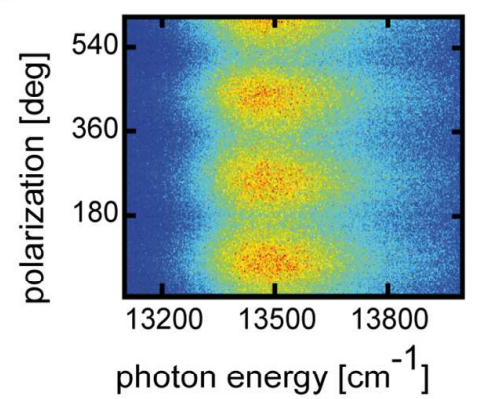

b)

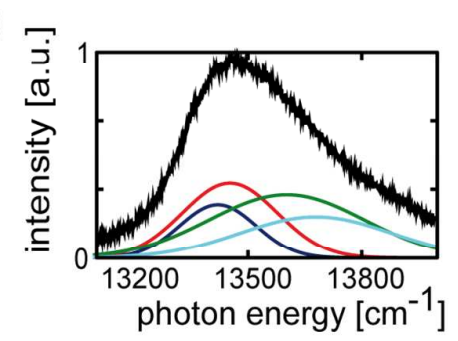

c)

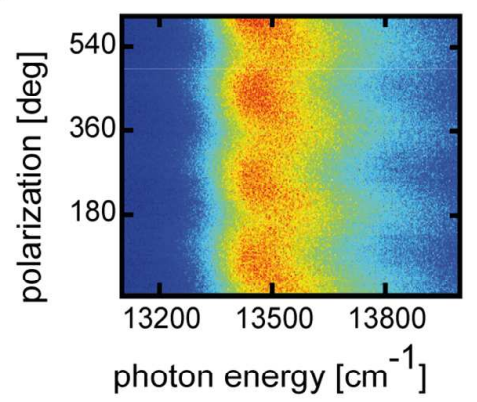

d)

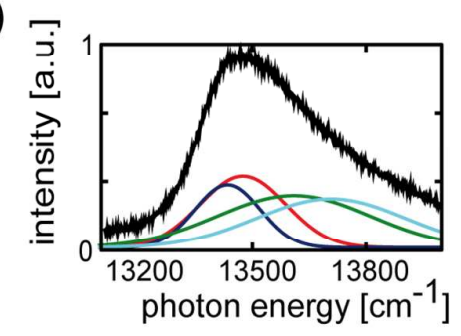

e)

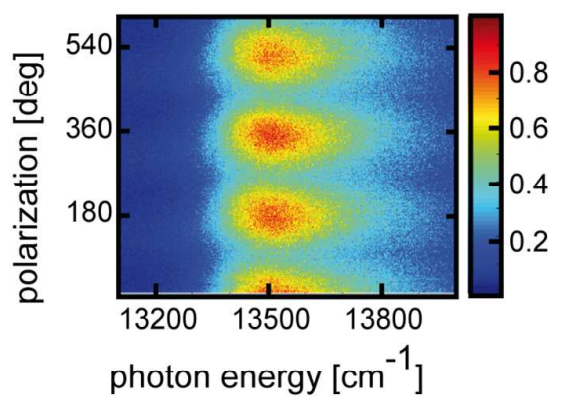

f)

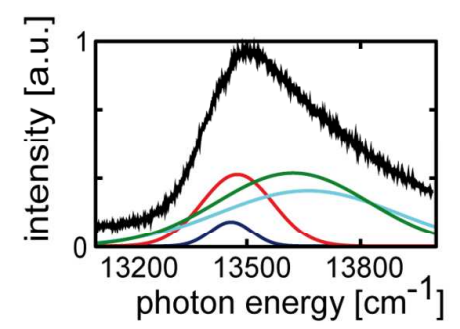

Figure S2: Examples of polarisation-resolved fluorescence-excitation spectra from three different individual chlorosomes, a,b; c,d; and e,f; respectively. The top panels $(\mathrm{a}, \mathrm{c}, \mathrm{e})$ correspond each to a stack of 250 fluorescenceexcitation spectra from one particular chlorosome as a function of the polarization of the excitation light. The spectra have been recorded at $1.5 \mathrm{~K}$. At the bottom $(b, d, f)$ the black lines correspond to the fluorescence-excitation spectra averaged over all polarisations, and the coloured lines represent the decomposition into four Gaussians.

Table S1: Spectral peak positions (E), linewidths (W,(FWHM)), and oscillator strengths (O) that can be extracted from the example spectra shown in figure $\mathrm{S} 2$.

\begin{tabular}{|l|l|l|l|l|}
\hline & $\mathbf{E}_{\mathbf{1}}\left[\mathbf{c m}^{-1}\right]$ & $\mathbf{E}_{\mathbf{2}}\left[\mathbf{c m}^{-1}\right]$ & $\mathbf{E}_{\mathbf{3}}\left[\mathbf{c m}^{-1}\right]$ & $\mathbf{E}_{\mathbf{4}}\left[\mathbf{c m}^{-1}\right]$ \\
\hline fig. S2 a/b & 13427 & 13459 & 13609 & 13686 \\
\hline fig. S2 c/d & 13439 & 13479 & 13613 & 13711 \\
\hline fig. S2 e/f & 13483 & 13494 & 13677 & 13731 \\
\hline
\end{tabular}

\begin{tabular}{|l|l|l|l|l|}
\hline & $\mathbf{W}_{\mathbf{1}}\left[\mathbf{c m}^{-\mathbf{1}}\right]$ & $\mathbf{W}_{\mathbf{2}}\left[\mathrm{cm}^{-\mathbf{1}}\right]$ & $\mathbf{W}_{\mathbf{3}}\left[\mathrm{cm}^{-\mathbf{1}}\right]$ & $\mathbf{W}_{\mathbf{4}}\left[\mathrm{cm}^{-\mathbf{1}}\right]$ \\
\hline fig. S2 a/b & 239 & 296 & 483 & 474 \\
\hline fig. S2 c/d & 203 & 267 & 477 & 463 \\
\hline fig. S2 e/f & 243 & 235 & 468 & 414 \\
\hline
\end{tabular}

\begin{tabular}{|l|l|l|l|l|}
\hline & $\mathbf{O}_{1}$ [a.u.] & $\mathbf{O}_{\mathbf{2}}$ [a.u.] & $\mathbf{O}_{\mathbf{3}}$ [a.u.] & $\mathbf{O}_{\mathbf{4}}$ [a.u.] \\
\hline fig. S2 a/b & 37 & 59 & 88 & 53 \\
\hline fig. S2 c/d & 51 & 72 & 100 & 85 \\
\hline fig. S2 e/f & 37 & 68 & 89 & 66 \\
\hline
\end{tabular}




\section{Correlation between the Energy Splittings $\Delta \mathrm{E}_{12}$ and $\Delta \mathrm{E}_{34}$}

The correlation between the energetic separations $\Delta \mathrm{E}_{\mathrm{ij}}$ and $\Delta \mathrm{E}_{\mathrm{kl}}$ (for $\mathrm{i}, \mathrm{j} \neq \mathrm{k}, \mathrm{l}$ ) of the spectral components from the same individual chlorosome is shown in fig.S3. A significant correlation is only observable for the energy splittings $\Delta \mathrm{E}_{12}$ vs. $\Delta \mathrm{E}_{34}$ of the spectral components within the low- and the high-energy doublet, fig.S3a.

a)

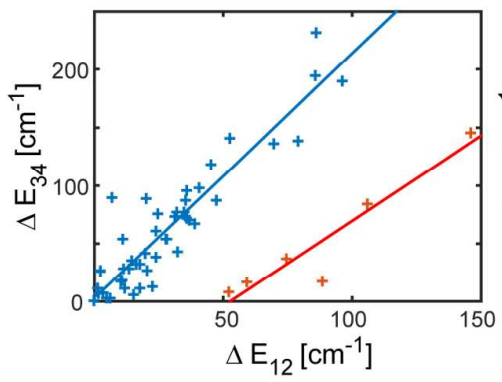

b)

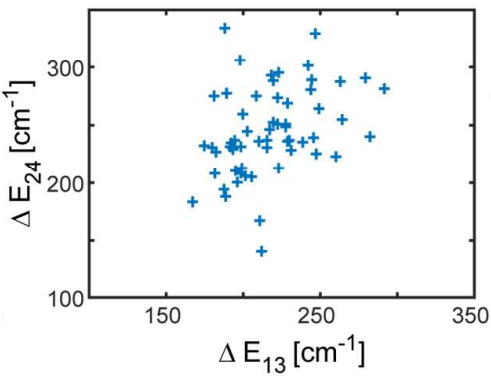

c)

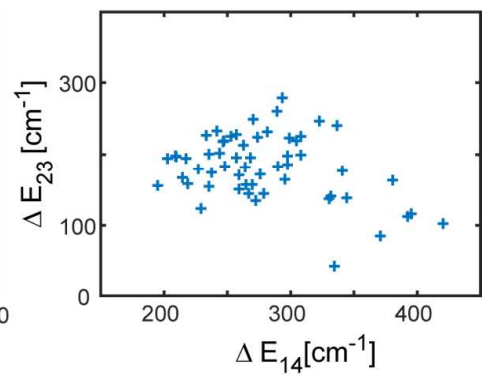

Figure S3: Each cross corresponds to the energetic separations $\Delta \mathrm{E}_{\mathrm{ij}}$ and $\Delta \mathrm{E}_{\mathrm{kl}}$ (for $\mathrm{i}, \mathrm{j} \neq \mathrm{k}, \mathrm{l}$ ) of the spectral components from the same individual chlorosome. a) $\Delta \mathrm{E}_{12} \mathrm{vs} . \Delta \mathrm{E}_{34}$, i.e. between the spectral components within the low- and the high-energy doublet. The correlation coefficient for the full data set from 58 individual chlorosomes amounts to 0.7. If the data points marked in blue or red are treated separately the correlation coefficients are 0.93 (blue) and 0.95 (red), respectively. Both relations are reasonably well represented by a linear dependence. b) $\Delta \mathrm{E}_{13} \mathrm{vs}$. $\Delta \mathrm{E}_{24}$. The correlation coefficient amounts to 0.36 . c) $\Delta \mathrm{E}_{14} \mathrm{vs} . \Delta \mathrm{E}_{23}$. The correlation coefficient amounts to 0.34 .

Two example spectra from chlorosomes that give rise to the data points marked in red in Fig. S3a are shown in Fig. S4. The setup of the figure is similar to that of Fig. S2. The fluorescence excitation spectra of these chlorosomes showed "unusual" features compared to the spectra from the other chlorosomes. For some of them the emitted intensity drops to a low level during data acquisition, Fig. S4a,b, whereas others display a pronounced shoulder on the high-energy side of the spectrum, Fig. S4c,d. Some of these chlorosomes exhibit both in their spectra: a drop in intensity as well as a pronounced high-energy wing. In particular the high-energy spectral feature is tempting us to speculate whether the data points marked in blue and red in Fig. S3a result from to two structural elements that differ quantitatively in their pigment arrangement. However, at the current stage this remains an open question. 
a)

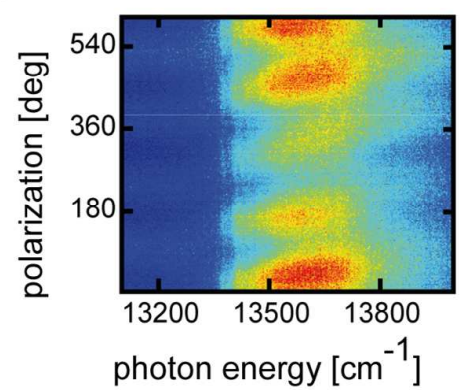

b)

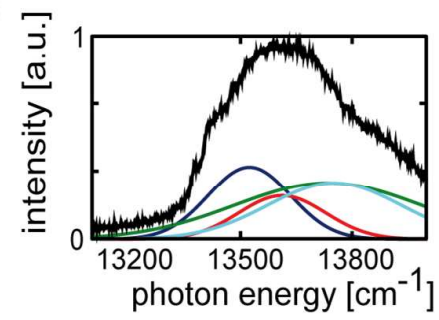

c)

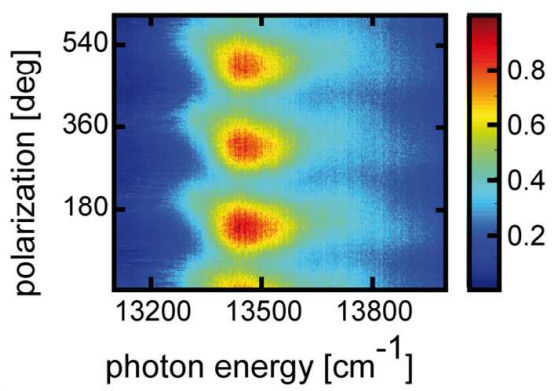

d)

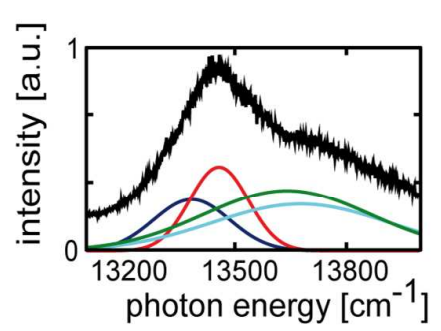

Fig. S4: Examples of spectra from individual chlorosomes that give rise to the data points marked in red in Fig. S3. The top panels $(a, c)$ correspond each to a stack of 250 fluorescence-excitation spectra from one particular chlorosome as a function of the polarization of the excitation light. The spectra have been recorded at $1.5 \mathrm{~K}$. At the bottom $(b, d)$ the black lines correspond to the fluorescence-excitation spectra averaged over all polarisations, and the coloured lines represent the decomposition into four Gaussians. 


\section{Cryo-electron microscopy}

Cryo-electron micrographs of $b c h R$ chlorosomes show that they look rather similar to WT chlorosomes. The lamellar organization, indicated by the striations in the direction of the long axis, appears somewhat less regular, suggesting more structural heterogeneity at the microscopic level (Fig. S5a). Images of individual chlorosomes were analysed by Fourier transformation of boxed areas. In the derived power spectra equatorial reflections are visible, originating from the lamellar spacing (green arrow in Fig. S5b). Although more fuzzy, the average lamellar spacing of $2.1 \mathrm{~nm}$ was the same as that found for the WT chlorosomes. A weak but significant layer line is present at $1 / 1.24 \mathrm{~nm}^{-1}$ (red arrow), suggesting a helical arrangement of the BChls in which hetero (syn-anti) dimers are the building blocks of the chlorophyll stacks, again similar to WT chlorosomes. 16 power spectra as in Fig. S5b were added after rotational alignment to illustrate the periodicity more clearly in Fig. 3a.
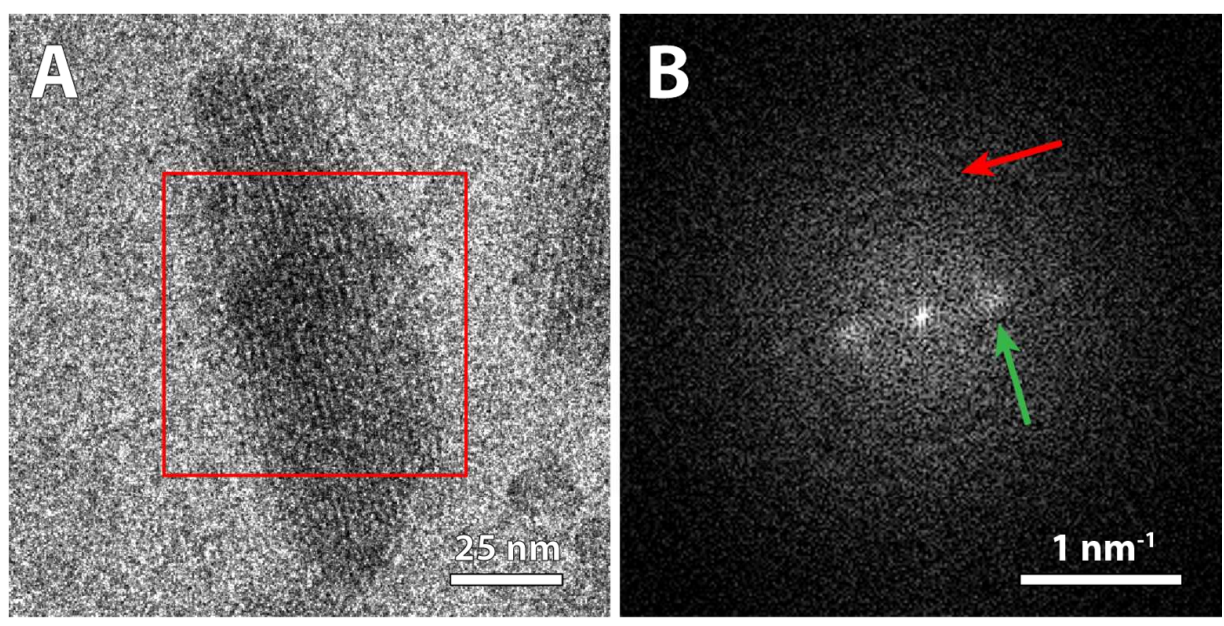

Fig. S5: (A) Cryo-electron micrograph of a bchR mutant chlorosome. (B) Power spectrum of the boxed area in (A). A green arrow points at the equatorial reflection originating from the lamellar spacing. The red arrow indicates the position of a layer line at $1 / 1.24 \mathrm{~nm}^{-1}$. 


\section{Simulations / Model Calculations}

a) Model structure

As a starting point for the simulations we used a planar oblique Bravais lattice as shown in Fig. $3 \mathrm{c}$ and Fig. S6.

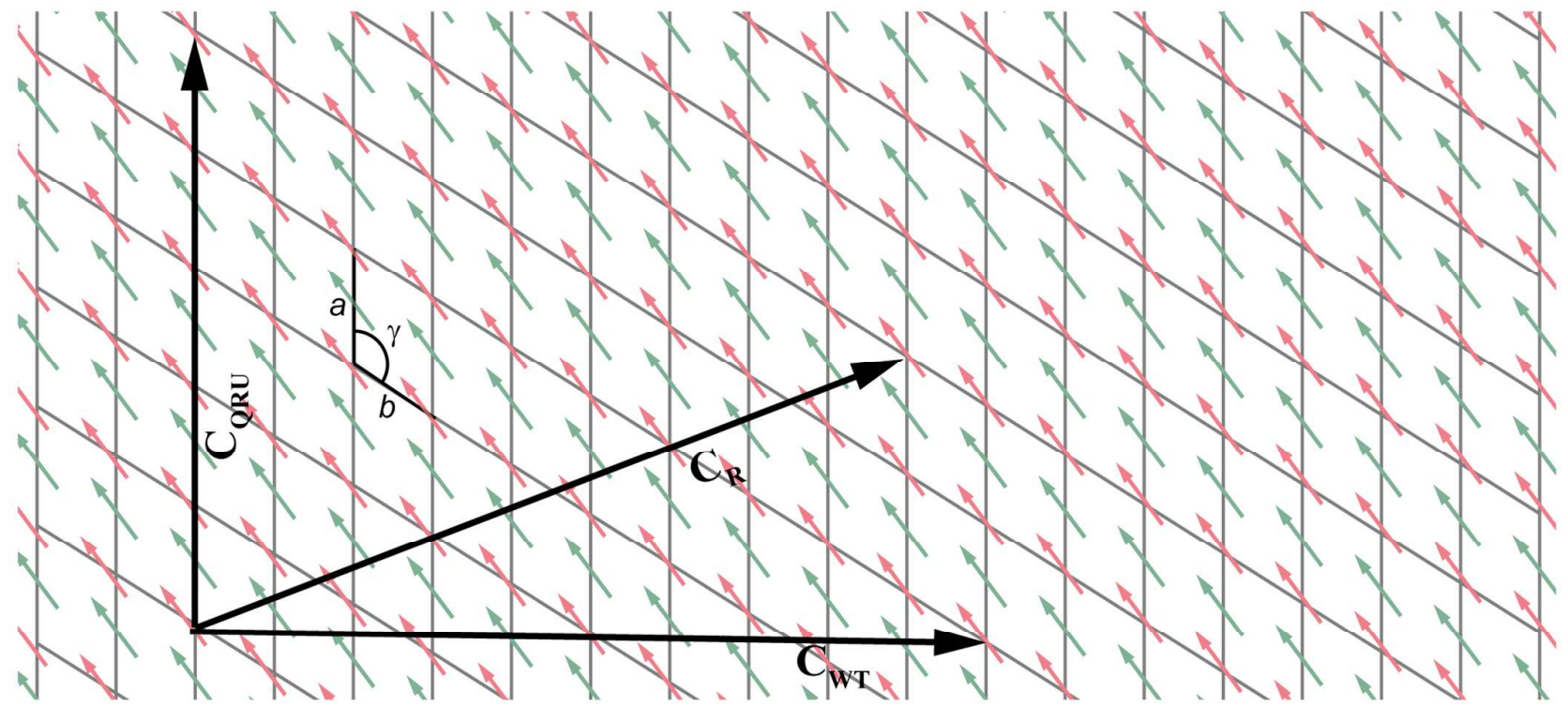

Figure S6: Bravais-lattice with lattice vectors of length $a=1.25 \mathrm{~nm}$ and $b=0.98 \mathrm{~nm}$ enclosing an angle of $\gamma=122^{\circ}$ for the arrangement of the $\mathrm{BChl}$ molecules as proposed $\mathrm{in}^{1}$. The vectors $\mathbf{C}$ refer to the rolling vector for the model structures proposed for the wild type ( $\mathbf{C}_{\mathrm{WT}}$ perpendicular to the $a$-axis), the bchQRU mutant ( $\mathbf{C}_{\mathrm{QRU}}$ parallel to the $a$ axis) and the $b c h R$ mutant ( $C_{R}$ enclosing an angle $\delta=69^{\circ}$ with the $a$-axis, this work).

This corresponds to the short-range order arrangement of the BChl molecules in WT chlorosomes from Cba. tepidum as reported in ${ }^{1}$. For the current study this lattice was wrapped under the rolling angle $\delta \approx 69^{\circ}$ onto a cylinder, where $\delta$ refers to the angle between the $a$-axis and the rolling vector $\mathbf{C}$, whose length is related to the cylinder radius: $|\mathbf{C}|=2 \pi R$. This yields a spacing between successive layers of $\mathrm{BChl}$ molecules of $\mathrm{d}=a \cdot \sin \delta$. The position of each monomer on the cylinder is given by:

$$
\mathrm{r}_{\mathrm{n}}=\left(r \cos \left(n_{1} \Phi_{1}+n_{2} \Phi_{2}\right), r \sin \left(n_{1} \Phi_{1}+n_{2} \Phi_{2}\right), n_{1} h\right)
$$

Here $r$ is the radius of the cylinder, $n=(n 1, n 2)$, with $n_{1}$ counting the rings, $\Phi_{1}$ is the azimuthal angle between adjacent rings in the stack, $n_{2}$ indicating the number of the monomer on the ring, and $\Phi_{2}$ is 
the azimuthal angle between adjacent monomers within a ring. The distance between successive rings is denoted by $\mathrm{h}$. The orientation of the corresponding transition-dipole moments is then given by:

$$
\vec{\mu}(n)=\left(\mu \sin (\beta) \sin \left(n_{1} \Phi_{1}+n_{2} \Phi_{2}-\alpha\right), \mu \sin (\beta) \cos \left(n_{1} \Phi_{1}+n_{2} \Phi_{2}-\alpha\right), \mu \cos (\beta)\right)
$$

for the monomers with a transition-dipole moment pointing outward with respect to the surface of the cylinder and

$$
\vec{\mu}(n)=\left(\mu \sin (\beta) \sin \left(n_{1} \Phi_{1}+n_{2} \Phi_{2}+\alpha\right), \mu \sin (\beta) \cos \left(n_{1} \Phi_{1}+n_{2} \Phi_{2}+\alpha\right), \mu \cos (\beta)\right)
$$

for the monomers with a transition-dipole moment pointing inward with respect to the surface of the cylinder. The angle $\alpha\left(=4^{\circ}\right)$ refers to the angle of the projection dipole with the local tangent of the ring (syn/anti stacking), and $\beta$ is the angle of the transition dipole with the cylinder axis ${ }^{2,3}$.

b) Model Hamiltonian

We have used the standard Frenkel exciton Hamiltonian in the Heitler-London approximation:

$$
H=\sum_{n=1}^{N} E_{0}|n\rangle\left\langle n\left|+\frac{1}{2} \sum_{n=1}^{N} \sum_{n \neq m} V_{n m}\right| n\right\rangle\langle m|
$$

where $|n\rangle$ indicates the state with molecule $n$ excited and all others in the ground state, $E_{0}$ corresponds to the transition energy of a monomer and $V_{n m}$ is the excitation transfer interaction between molecules $n$ and $m$. The latter was calculated in the point-dipole approximation

$$
V_{n m}=\frac{\vec{\mu}_{n} \cdot \vec{\mu}_{m}}{\left|\vec{r}_{n m}\right|^{3}}-3 \frac{\left(\vec{\mu}_{n} \cdot \vec{r}_{n m}\right)\left(\vec{\mu}_{m} \cdot \vec{r}_{n m}\right)}{\left|\vec{r}_{n m}\right|^{5}}
$$

Here $\vec{\mu}_{n}$ and $\vec{\mu}_{m}$ correspond to the transition-dipole moments of molecules $n$ and $m$, and $\vec{r}_{n m}$ refers to their relative position vector.

c) Model spectra

The Hamiltonian for a particular cylinder was diagonalized, which yielded the collective excited states of the model chlorosome in the form $|k\rangle=\sum_{n} \Phi_{k n}|n\rangle$ and their energies $E_{k}$. Here $k$ labels the various eigenstates and $E_{k}$ and $\phi_{\mathrm{kn}}$ are obtained as eigenvalues and eigenvectors, respectively, of the matrix with as diagonal elements the molecular transition energies $E_{0}$ and as off-diagonal elements the interactions $V_{n m}$. The fluorescence excitation spectrum was then obtained as

$$
F E(\omega)=S_{k}^{-1} \sum_{k}\left|\mu_{k} \cdot e\right|^{2} \exp \left[-\left(\hbar \omega-E_{k}\right)^{2} / 2 S_{k}^{2}\right]
$$


where $S_{k}$ is the inhomogeneous width attributed to exciton state $k$ expressed as a standard deviation, which can be converted into a FWHM according to $W_{k}=2 \sqrt{2 \ln 2} S_{k}$, e indicates the polarization vector of the exciting light, and $\mu_{k}=\sum_{n} \Phi_{k n} \mu(n)$ indicates the transition dipole vector between the chlorosome's ground state and dipole and its $k^{\text {th }}$ exciton state.

\section{d) Model Parameters}

The simulations have been carried out for cylindrical structures of different radii that have a length of $30 \mathrm{~nm}$ each and $\mathrm{N}$ molecules. In order to obtain closed cylinders that are consistent with the results from previous NMR and cryo-EM experiments, previous molecular modelling for the WT and QRU mutant structures, as well as with the results from cryo-EM and optical experiments obtained in this work, the lattice parameters of the grid that determined the short-range order were slightly varied as detailed in Table S2.

Table S2: Parameters used to simulate the spectrum presented in fig. $4 a$.

\begin{tabular}{|l|l|l|l|l|l|l|l|l|l|l|}
\hline & $\mathrm{a}[\mathrm{nm}]$ & $\mathrm{b}[\mathrm{nm}]$ & $\mathrm{d}[\mathrm{nm}]^{* *}$ & $\mathrm{r}[\mathrm{nm}]$ & $\delta\left[^{\circ}\right]$ & $\eta\left[^{\circ}\right]$ & $\beta\left[^{\circ}\right]^{*}$ & $\mu[\mathrm{D}]$ & $\begin{array}{l}\mathrm{E}_{0} / \mathrm{hc} \\
{\left[\mathrm{cm}^{-1}\right]}\end{array}$ & $\mathrm{N}$ \\
\hline Cylinder 1 & 1.297 & 0.98 & 1.21 & 14.7 & 69 & 35 & 56 & 5.5 & 15300 & 5152 \\
\hline Cylinder 2 & 1.294 & 0.98 & 1.24 & 9.7 & 79 & 35 & 52 & 5.5 & 15500 & 3388 \\
\hline
\end{tabular}

* The angle $\beta$, is directly linked to the rolling angle $\delta$ via $\beta=\left|\left(90^{\circ}-\delta+\eta\right) \bmod 180^{\circ}\right|$

** The stacking distance $\mathrm{d}$ along the tube's axis is given by $\mathrm{d}=a \cdot \sin \delta$.

We used the angle $\delta$ as the angle of the rolling vector. For the dipole strength of the monomers we used $\mu^{2}=30 D^{2}{ }^{4}$, and for the site energies of the monomers within the two cylinders we used 15300 $\mathrm{cm}^{-1}$ and $15500 \mathrm{~cm}^{-1}$, respectively. A slight difference in site energies can be the result of different local environments, caused for example by different dielectric shielding. This is not unlikely if both cylinders together make up a double-walled structure. 
a)

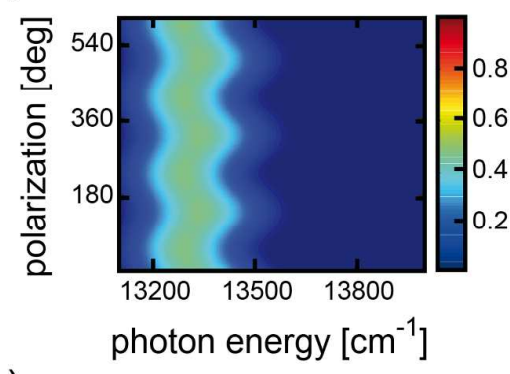

c)

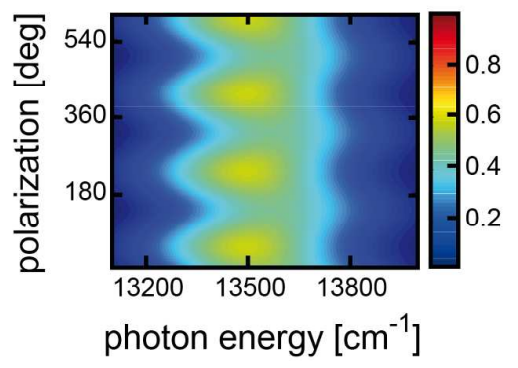

b)

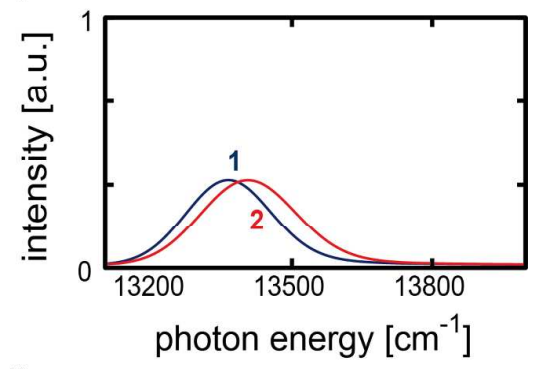

d)

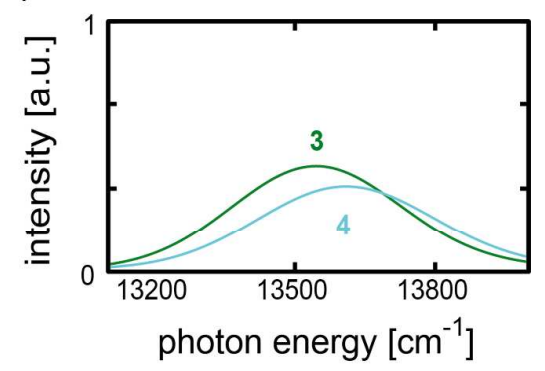

Figure S7: Spectra of the individual cylinders used for the simulation shown in Fig. 4a in the main text. a),b) Contributions from cylinder 1; polarisation-resolved (a), and averaged over all polarisations (b). c), d) Contributions from cylinder 2; polarisation-resolved (c), and averaged over all polarisations (d). The values used for the parameters of the simulation are given in Table S2. The colour code is equivalent to the colour code used for the figures in the main text.

Subsequently, the stick spectrum resulting from eigenvectors and eigenvalues of the Hamiltonian was dressed with a gaussian lineshapes. The width of the gaussians were chosen similar to the results from the experiment: $S_{1}=100 \mathrm{~cm}^{-1}, S_{2}=100 \mathrm{~cm}^{-1}, S_{3}=200 \mathrm{~cm}^{-1}, S_{4}=200 \mathrm{~cm}^{-1}$. Figs. $S 7$ a-d show the contributions of the individual cylinders to the overall spectrum shown in Fig. 4 a. 


\section{Variations of the model structure}

In order to address the widths of the distributions of the spectral parameters we calculated for a single-wall cylindrical aggregate the spectral positions and the intensities of the transitions as a function of the radius $r$, rolling angle $\delta$ and lattice parameter $a$ on the spectral positions and intensities of the perpendicular and parallel absorption bands. Therefore, we have chosen the values applied for one of the cylinder structures used for Fig. 4a as the default values (see Table S3). Subsequently, one of these parameters was varied within reasonable limits, while all other parameters were fixed at their default values, see Table S3. In particular we find that changing the radius of the cylinder, mainly affects the energy spacing between the superradiant transitions (Fig. S8a). Furthermore, small changes of the rolling direction of the lattice, i.e. the angle $\delta$, affect the ratio of the oscillator strengths of the parallel and perpendicular bands (Fig. S8b). Finally, the unit cell parameter $a$, which is directly related to $\delta$ via $d=a \cdot \sin \delta$, influences the spectral position of both bands (Figs. S8c). These calculations show that broadening of the Gaussian contributions to the overall spectrum as well as the fluctuations seen in the histograms of Fig. 2 might be caused by small variations of the molecular arrangement and sizes of the tubes.

a)

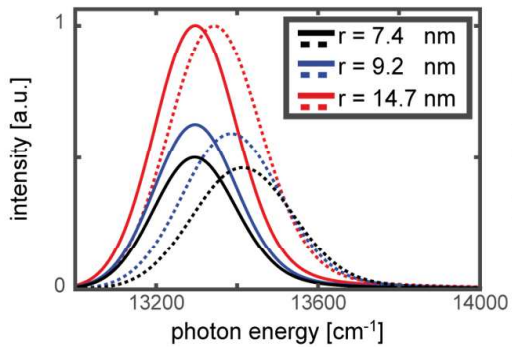

b)

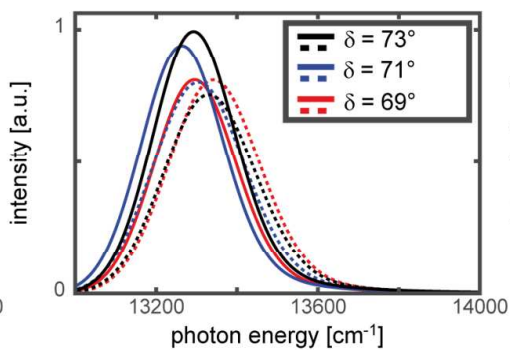

c)

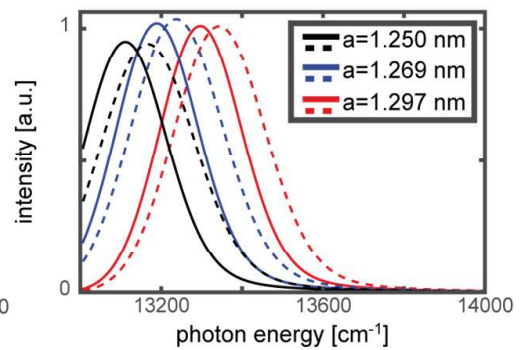

Figure S8: Simulated spectra from cylinder structures based on the model described above and in the main text. Each panel shows spectra for three cylinders that differ with respect to each other in one of the model parameters. (a) variation of the cylinder radius $r$, (b) variation of the wrapping angle $\delta$, (c) variation of the lattice parameter $a$ of the unit cell. In the individual panels the solid line corresponds to the spectral component polarized parallel to the cylinder axis, and the dashed line corresponds to the spectral component polarized perpendicular to the cylinder axis, whereas different colours refer to different values of the parameters used. 
Table S3: Model parameters used to simulate the spectra displayed in figure S8. The colours in the legend on the left hand side correspond to the colours of the spectra shown in the figure.

\begin{tabular}{|l|l|l|l|l|l|l|l|}
\hline \multicolumn{6}{|l|}{ Set of default values of cylinder 1 in fig. S7 } & \\
\hline & $a[\mathrm{~nm}]$ & $d[\mathrm{~nm}]^{*}$ & $r[\mathrm{~nm}]$ & $\delta\left[^{\circ}\right]$ & $\eta\left[^{\circ}\right]$ & $\beta\left[^{\circ}\right]$ & $\mathrm{N}$ \\
\hline Red & 1.297 & 1.21 & 14.7 & 69 & 35 & 56 & 5152 \\
\hline Variation of radius $r$ (fig.S8 a) & 1.297 & 1.21 & 9.2 & 69 & 35 & 56 & 3220 \\
\hline Blue & 1.297 & 1.21 & 7.4 & 69 & 35 & 56 & 2576 \\
\hline Black & Variation of rolling angle $\delta^{* *}$ (fig. S8 b) \\
\hline Blue & 1.287 & 1.22 & 15.4 & 71 & 35 & 54 & 5400 \\
\hline Black & 1.297 & 1.24 & 15.2 & 73 & 35 & 52 & 5324 \\
\hline Variation of lattice parameter a (fig. S8 c) & 14.8 & 69 & 35 & 56 & 5280 \\
\hline Blue & 1.269 & 1.18 & 13.5 & 69 & 35 & 56 & 4890 \\
\hline Black & 1.250 & 1.17 &
\end{tabular}

* The stacking distance $\mathrm{d}$ is given by $\mathrm{d}=a \cdot \sin \delta$.

**As mentioned in the main text, for the mutual relationship of the angles $\beta, \delta$, and $\eta$ holds the relation $\beta=\left|\left(90^{\circ}-\delta+\eta\right) \bmod 180^{\circ}\right|$. For the simulations where we varied $\delta$ we fixed the value of $\eta$ at $35^{\circ}$ for better comparison and adjusted $\beta$ according to this expression.

It is interesting to investigate the influence of the radius of the cylinder on the energetic separation $\Delta E_{\|, \perp}$ of the two mutually orthogonal polarised spectral components. (In the main text $\Delta E_{\|, \perp}$ is referred to as $\Delta \mathrm{E}_{12}$ or $\Delta \mathrm{E}_{34}$, respectively.) This splitting increases for increasing curvature, i.e. decreasing radius of the underlying cylinder, see Table S4 and Fig. S9. For a single chlosorome that accommodates several cylindrical structures of varying radii, this might be the mechanism that contributes predominantly to the inhomogeneous line broadening.

Table S4: Relation between the radius of the underlying cylinder structure and the energetic separation $\Delta E_{\|, \perp}$ of the two mutually orthogonal polarised bands in the spectrum.

\begin{tabular}{|l|l|l|l|l|l|l|l|l|}
\hline$r[\mathrm{~nm}]$ & 3.7 & 5.5 & 7.4 & 9.2 & 11.1 & 12.9 & 14.7 & 16.6 \\
\hline$\Delta E_{\|, \perp}\left[\mathrm{cm}^{-1}\right]$ & 270 & 169 & 119 & 89 & 70 & 57 & 47 & 40 \\
\hline
\end{tabular}




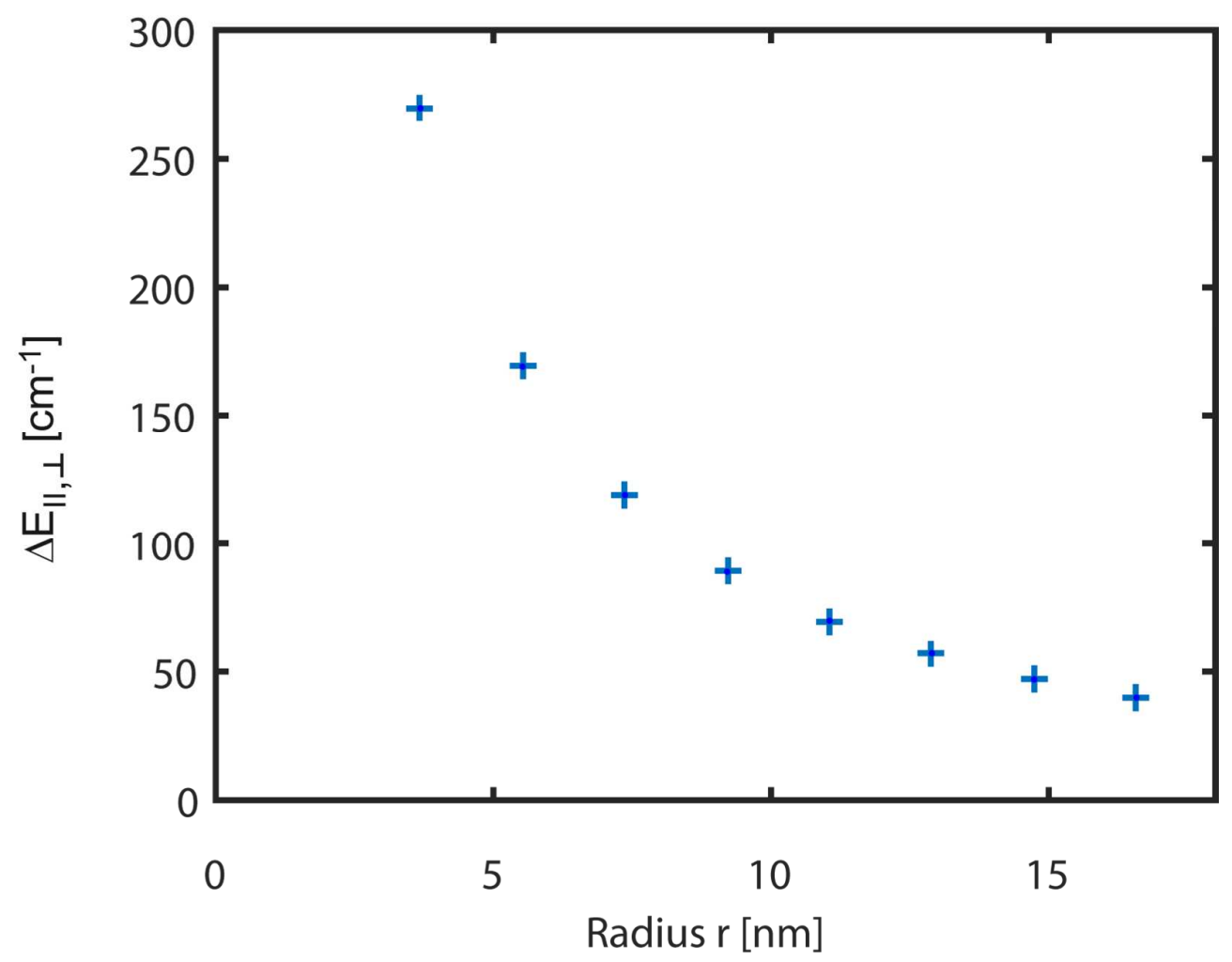

Figure S9: Energetic separation, $\Delta E_{\|, \perp}$, between the two mutually orthogonal polarised spectral components in a doublet as a function of the cylinder radius. In the main text these spectral splittings are referred to as $\Delta \mathrm{E}_{12}$ and $\Delta \mathrm{E}_{34}$, respectively. For this example calculation the following parameters have been used: $\delta=69^{\circ}, \eta=35^{\circ}, a=1.297 \mathrm{~nm}, b=0.98 \mathrm{~nm}$

\section{References}

(1) Ganapathy, S.; Oostergetel, G. T.; Wawrzyniak, P. K.; Reus, M.; Gomez Maqueo Chew, A.; Buda, F.; Boekema, E. J.; Bryant, D. A.; Holzwarth, A. R.; de Groot, H. J. M. Alternating Syn-Anti Bacteriochlorophylls Form Concentric Helical Nanotubes in Chlorosomes. Proc. Natl. Acad. Sci. 2009, 106 (21), 8525-8530.

(2) Didraga, C.; Klugkist, J. A.; Knoester, J. Optical Properties of Helical Cylindrical Molecular Aggregates: The Homogeneous Limit. J. Phys. Chem. B 2002, 106 (44), 11474-11486.

(3) Didraga, C.; Pugžlys, A.; Hania, P. R.; von Berlepsch, H.; Duppen, K.; Knoester, J. Structure, Spectroscopy, and Microscopic Model of Tubular Carbocyanine Dye Aggregates. J. Phys. Chem. $B$ 2004 Sep 30, 108 (39), 14976-14985.

(4) Prokhorenko, V. I.; Steensgaard, D. B.; Holzwarth, A. R. Exciton Dynamics in the Chlorosomal Antennae of the Green Bacteria Chloroflexus aurantiacus and Chlorobium tepidum. Biophys. J. 2000, 79 (4), 2105-2120. 